\title{
Sub-2 nm Size-Tunable High-Density Pt Nanoparticle Embedded Nonvolatile Memory
}

\author{
Minseong Yun, David W. Mueller, Student Member, IEEE, Maruf Hossain, Veena Misra, Senior Member, IEEE, \\ and Shubhra Gangopadhyay, Senior Member, IEEE
}

\begin{abstract}
The charge-storage characteristics of a metal-oxidesemiconductor (MOS) structure containing size-tunable sub-2 nm Pt nanoparticles (NPs) between $\mathrm{Al}_{2} \mathrm{O}_{3}$ tunneling and capping oxide layers were studied. Significantly different amounts of memory window were obtained with the different sizes of Pt NP embedded MOS structures and reached a maximum of $4.3 \mathrm{~V}$ using a $1.14 \mathrm{~nm}$ Pt NP, which has the strongest charging capability caused by optimum size and the largest particle density obtained in our deposition method. Satisfactory long-term nonvolatility was attained in a low electric field due to the Coulomb blockade and quantum confinement effects in $\sim 1 \mathrm{~nm}$ Pt NP. These properties are very promising in view of device application.
\end{abstract}

Index Terms-Nanoparticle (NP), nonvolatile memory (NVM), size-tunable platinum.

\section{INTRODUCTION}

$\mathbf{N}$ ONVOLATILE memory (NVM) devices using chargestorage mechanisms face serious obstacles due to the further downscaling of tunneling oxide thickness [1]. Metal nanoparticle (NP) embedded NVM devices have been attractive because metal has a larger work function and higher density of states than a semiconductor [2], [3] and it is easy to tune the barrier height for carrier injection due to numerous choices of metal [4]. In addition, metal NP embedded NVMs employing high- $k$ dielectrics enable equivalent oxide thickness scaling and thus exhibit smaller operating voltage, faster program/erase (P/E) speeds, better data endurance, and long retention characteristics [5]. NPs smaller than $2 \mathrm{~nm}$ have the added benefit of the Coulomb blockade effect [6].

There are several ways to form metal NPs using physical vapor deposition. Specific to Pt NPs, two examples of NP formation processes include thermal dewetting technique [7], [8] and electron beam evaporation [9]. The first technique does not satisfy a low thermal budget and the particle distributions are broad, and the second technique shows inability to control uniform and spherically shaped NPs smaller than $5 \mathrm{~nm}$.

Manuscript received July 20, 2009; revised September 23, 2009. Current version published November 20,2009. This work was supported in part by the National Science Foundation under Grant GOALI-0523656 and in part by Techguard Security, LLC. The review of this letter was arranged by Editor T. Wang.

M. Yun, D. W. Mueller, M. Hossain, and S. Gangopadhyay are with the Department of Electrical and Computer Engineering, University of Missouri, Columbia, MO 65211 USA (e-mail: mynn3@mail.missouri.edu; dwm83a@ mail.missouri.edu; hossainma@missouri.edu; gangopadhyays@missouri.edu).

V. Misra is with the Department of Electrical and Computer Engineering, North Carolina State University, Raleigh, NC 27695 USA (e-mail: vmisra@ncsu.edu).

Digital Object Identifier 10.1109/LED.2009.2033618
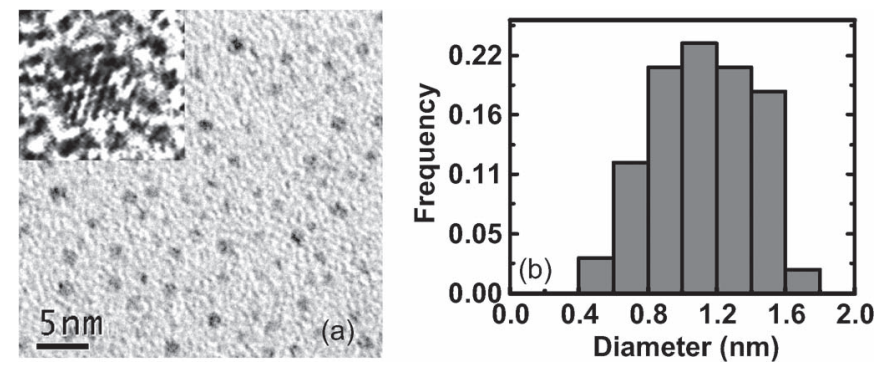

Fig. 1. (a) Plane-view TEM images of $20 \mathrm{~s}$ deposited Pt NPs. The inset of (a) shows monocrystallinity of Pt NPs. (b) Particle size distribution of $20 \mathrm{~s}$ deposited Pt NP samples over three images after statistical postimage analysis. The error in the analysis is caused by the lack of contrast between the NPs and the amorphous substrate material.

Recently, we have conducted research on metal NP formation using sputtering deposition without subsequent annealing. In this direct deposition, the average size of the NPs is simply controlled by varying deposition time with constant pressure, power, and gas flow rate at room temperature. This technique produces uniformly distributed spherical Pt NPs with mean diameters between 0.5 and $2 \mathrm{~nm}$, having a high particle density $>10^{12} \mathrm{~cm}^{-2}$. In this method, we exploit the earliest stages of film growth where metal clusters arrive on the substrate surface and diffuse until encountering nucleation (trap) sites [10], [11]. Small clusters remain trapped at these sites and grow in size due to the continuous flux of atoms. The large surface energy difference between $\mathrm{Al}_{2} \mathrm{O}_{3}\left(40-50 \mathrm{~mJ} / \mathrm{m}^{2}\right)$ and $\mathrm{Pt}$ $\left(2190 \mathrm{~mJ} / \mathrm{m}^{2}\right)$ dictates a Volmer-Weber type islanding growth mode with spherical particles. Our major motivation for a sub$2 \mathrm{~nm}$ size-tunable metal NP together with high particle density is for better controllability of the different memory capabilities of scaled NVM devices [12].

In this letter, we demonstrate size-tunable sub-2 nm Pt NP embedded metal-oxide-semiconductor (MOS) devices, utilizing thin $\mathrm{Al}_{2} \mathrm{O}_{3}$ tunneling and control oxide layers. Endurance and retention characteristics are also demonstrated.

\section{Device FABRicAtion}

Low-doped p (100) silicon was used to fabricate MOS capacitors. An e-beam system was utilized to deposit a $4.3 \mathrm{~nm}$ tunneling $\mathrm{Al}_{2} \mathrm{O}_{3}$ layer. Samples were immediately transferred to the sputtering system to deposit Pt NPs onto the tunneling oxide with the following deposition times: 5, 10, 20, 30, and $45 \mathrm{~s}$. A power of $30 \mathrm{~W}$ RF was used with a working pressure of $4 \mathrm{mTorr}$ and $10 \mathrm{sccm}$ of Ar gas flown at room temperature 
TABLE I

Summary of Platinum NPs as a Function of Deposition Time

\begin{tabular}{|c|c|c|c|c|c|c|}
\hline $\begin{array}{l}\text { Deposition } \\
\text { time }(\mathrm{s})\end{array}$ & $\begin{array}{l}\text { Nominal } \\
\text { thickness }(\mathrm{nm})\end{array}$ & $\begin{array}{l}\text { Average } \\
\text { size }(n m)\end{array}$ & $\begin{array}{c}\text { Average particle } \\
\text { density }\left(\times 10^{12} \mathrm{~cm}^{-2}\right)\end{array}$ & $\begin{array}{c}\text { Memory } \\
\text { window (V) }\end{array}$ & $\begin{array}{l}\text { Electron charge density } \\
\qquad\left(\times 10^{12} \mathrm{~cm}^{-2}\right)\end{array}$ & $\begin{array}{c}\text { Number of } \\
\text { electrons per NP }\end{array}$ \\
\hline 5 & $\sim 0.002$ & $\sim 0.5$ & $\sim 1-3$ & 0.92 & 1.1 & $0.36-1.1$ \\
\hline 10 & $\sim 0.011$ & $0.8 \pm 0.1$ & $3.95 \pm 1.08$ & 2.03 & 2.3 & 0.58 \\
\hline 20 & $\sim 0.038$ & $1.14 \pm 0.07$ & $4.87 \pm 0.63$ & 4.26 & 4.7 & 0.97 \\
\hline 30 & $\sim 0.094$ & $1.54 \pm 0.05$ & $4.9 \pm 0.85$ & 3.78 & 4.3 & 0.77 \\
\hline 45 & $\sim 0.200$ & $1.98 \pm 0.02$ & $4.85 \pm 0.2$ & 1.63 & 1.9 & 0.39 \\
\hline
\end{tabular}
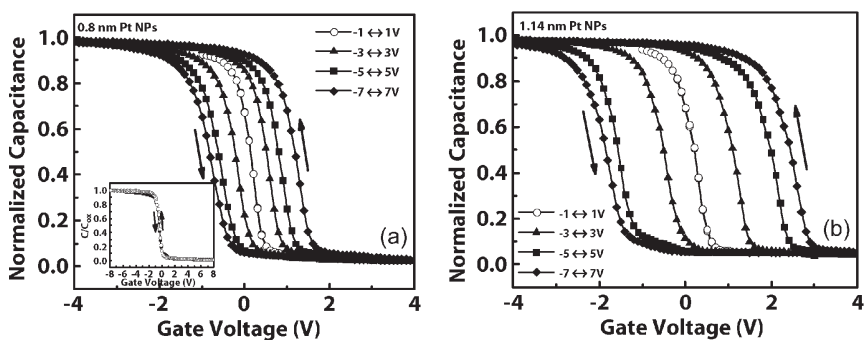

Fig. 2. High-frequency $(1 \mathrm{MHz})$ bidirectional $C-V$ curves of (a) 0.8 and (b) $1.14 \mathrm{~nm}$ Pt NP embedded memory device under different sweeping gate voltages. The $C-V$ curve of the control sample is shown in the inset of (a). The control and active devices have negligible hysteresis when uncharged, as shown in (open circles) the initial voltage sweep. The $V_{\mathrm{FB}}$ of this range is the initial $V_{\mathrm{FB}}$ and used for $V_{\mathrm{FB}}$ shift calculation. The left three branches represent hole charging states while the right three branches represent electron charging states.

[10]. Samples were again transferred to the e-beam for $14 \mathrm{~nm}$ $\mathrm{Al}_{2} \mathrm{O}_{3}$ capping oxide deposition and subsequent in situ $\mathrm{H}_{2}$ gas annealing at $260{ }^{\circ} \mathrm{C}$ for $45 \mathrm{~min}$. Finally, $250 \mathrm{~nm}$ Ti electrodes of $4.42 \times 10^{-5} \mathrm{~cm}^{2}$ were patterned using a shadow mask. A control sample without NPs was also prepared by the same process. Capacitance-voltage $(C-V)$ measurements were performed using a Keithley 4200-SCS equipped with the 4200-CVU integrated $C-V$ option. Data were taken with a voltage step of $0.1 \mathrm{~V}$ and a $30 \mathrm{mV}$ ac signal at $1 \mathrm{MHz}$.

\section{RESULTS AND DISCUSSION}

Fig. 1 shows the plane-view transmission electron microscopy (TEM) image (a) and size-distribution analysis (b) of 20 s deposited Pt NPs, whose average size and density are estimated as $1.14 \mathrm{~nm}$ and $4.87 \times 10^{12} \mathrm{~cm}^{-2}$, respectively. The highresolution TEM image in the inset of Fig. 1(a) shows the monocrystalline structure of the Pt NP. Table I summarizes the nominal layer thickness, size, and density of Pt NPs according to the different deposition times.

Fig. 2(a) and (b) shows the $C-V$ curves of 0.8 - and $1.14 \mathrm{~nm}$ Pt NP embedded memory devices under different sweeping voltages. The inset of Fig. 2(a) shows that there is a negligible hysteresis in control sample under the same applied voltage. Thus, the $V_{\mathrm{FB}}$ shift of the active device is attributed to electron charging in Pt NP or at the interface between NP and $\mathrm{Al}_{2} \mathrm{O}_{3}$, not from the defects in $\mathrm{Al}_{2} \mathrm{O}_{3}$ [13]. Counterclockwise hysteresis loops were observed, indicating electron injection from substrate to Pt NPs under positive voltage for the programming and hole injection under negative voltage for erasing by Fowler-Nordheim (F-N) tunneling [7]. It was also shown that $V_{\mathrm{FB}}$ shift under different sweeping voltages gradually increased

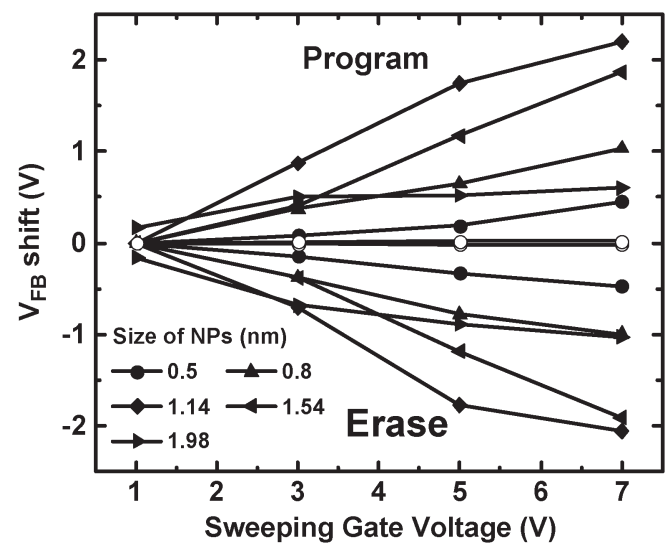

Fig. 3. $\quad V_{\mathrm{FB}}$ shift as a function of $\mathrm{P} / \mathrm{E}$ voltages from $C-V$ curves according to the different sizes of Pt NPs. (Light open and labeled) Negligible $V_{\mathrm{FB}}$ shift in the control samples is shown.

with a uniform increase of F-N injection and finally saturated over a certain value within the measurement range.

Interestingly, there was a remarkable difference of memory window between 0.8 - and $1.14 \mathrm{~nm}$ Pt NP embedded samples. The $V_{\mathrm{FB}}$ shift as a function of $\mathrm{P} / \mathrm{E}$ voltages according to the different size of Pt NPs is shown in Fig. 3. Initially, there was no memory effect in the control sample, but the $V_{\mathrm{FB}}$ shift increased gradually with increased size and density of Pt NPs. The memory window reached its maximum value of $4.3 \mathrm{~V}$ with the $1.14 \mathrm{~nm}$ Pt NP sample which has optimum size and maximum density of NP, resulting in the strongest charging capability among the samples. As the particle size increased, the memory window decreased due to a reduction in Coulomb charging energy [6]. However, the exact nature of the decreasing memory window is still not clear, and we are striving to find the reason using further experiments such as different speeds of $\mathrm{P} / \mathrm{E}$ characteristics and single electron tunneling effect. This will be presented as a follow-up paper. Since the $C-V$ curve between $\pm 1 \mathrm{~V}$ with no memory window is located approximately in the middle of the hysteresis loop for each device, the amount of electron storage in Fig. 3 will be half of the area of the hysteresis loop. By using the relationship $N_{t}=\left(C_{\mathrm{ox}} \Delta_{\mathrm{FB}}\right) / q$, where $N_{t}$ is the trapped charge, $C_{\mathrm{ox}}$ is the oxide capacitance density, $\Delta_{\mathrm{FB}}$ is the $V_{\mathrm{FB}}$ shift, and $q$ is the elementary charge, we find that, for $1.14 \mathrm{~nm}$ Pt NPs with particle density $\left(4.87 \times 10^{12} \mathrm{~cm}^{-2}\right)$ and electron charge density $\left(4.7 \times 10^{12} \mathrm{~cm}^{-2}\right)$ after programming, each Pt NP serves one electron storage node due to the small $\sim 1 \mathrm{~nm}$ size of NP, which is truly a single electron memory device.

As shown in Fig. 4(a), the endurance characteristics of the $1.14 \mathrm{~nm}$ Pt NP embedded memory devices were observed for up 

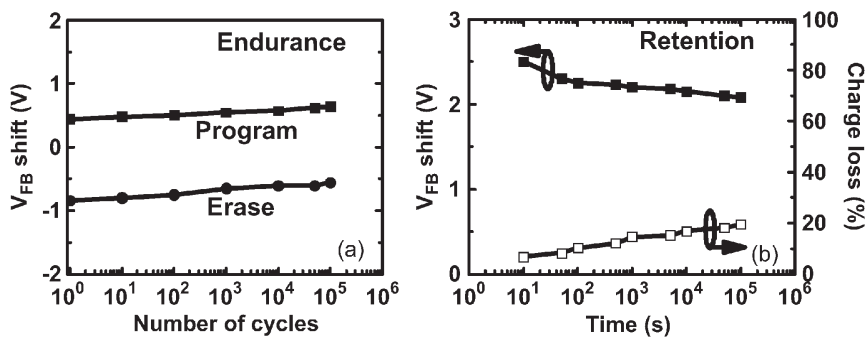

Fig. 4. (a) Endurance characteristic of $1.14 \mathrm{~nm}$ Pt NP embedded memory device. (b) Retention properties of the memory device at programming and its charge loss percentage versus time. The percentage of charge loss is calculated using the expression $\left(1-V_{\mathrm{FB}}(t) / V_{\mathrm{FB}}(0)\right) \times 100$, where $V_{\mathrm{FB}}(0)$ is the $V_{\mathrm{FB}}$ after stressing and $V_{\mathrm{FB}}(t)$ is the $V_{\mathrm{FB}}$ at the time of interest.

to $10^{5}$ cycles with $100 \mathrm{~ms}$ stress pulses of $\pm 6 \mathrm{~V}$, demonstrating a stable memory window. No significant memory narrowing was observed up to $10^{5}$ cycles. The memory device also showed good retention characteristics when applying a $5 \mathrm{~s}$ stress pulse of $7 \mathrm{~V}$ program voltage and sweeping $C-V$ periodically, as shown in Fig. 4(b). Initial capacitance abruptly dropped by $10 \%$ within a short period of time due to the tunneling back of electrons and then maintained its $V_{\mathrm{FB}}$ up to $10^{5} \mathrm{~s}$ because of the buildup of a high opposing electric field in the tunnel oxide.

\section{CONCLUSION}

Pt NPs using room-temperature sputter deposition with a simple variation of deposition time were embedded into $\mathrm{Al}_{2} \mathrm{O}_{3}$ double layers to develop NVM devices. The memory devices showed different amounts of memory window from 1 to $4.3 \mathrm{~V}$ under low P/E voltages, good endurance, and long retention properties without narrowing. Our approach in developing metal NP embedded NVM devices is a good candidate for nonvolatile Flash memory and is compatible with low-temperature semiconductor processing.

\section{REFERENCES}

[1] International Technology Roadmap for Semiconductors, 2007. [Online]. Available: http://public.itrs.net

[2] S. Tiwari, F. Rana, H. Hanafi, A. Hartstein, E. F. Crabbé, and K. Chan, "A silicon nanocrystals based memory," Appl. Phys. Lett., vol. 68, no. 10, pp. 1377-1379, Mar. 1996.

[3] D. Zhao, Y. Zhu, R. Li, and J. Liu, "Simulation of a Ge-Si heteronanocrystal memory," IEEE Trans. Nanotechnol., vol. 5, no. 1, pp. 37-41, Jan. 2006.

[4] Z. Liu, C. Lee, V. Narayanan, G. Pei, and E. C. Kan, "Metal nanocrystal memories-Part I: Device design and fabrication," IEEE Trans. Electron Devices, vol. 49, no. 9, pp. 1606-1613, Sep. 2002.

[5] J. J. Lee and D.-L. Kwong, "Metal nanocrystal memory with high- $\kappa$ tunneling barrier for improved data retention," IEEE Trans. Electron Devices, vol. 52, no. 4, pp. 507-511, Apr. 2005.

[6] H. Tsuji, N. Arai, T. Matsumoto, K. Ueno, Y. Gotoh, K. Adachi, H. Kotaki, and J. Ishikawa, "Silver nanoparticle formation in thin oxide layer on silicon by silver-negative-ion implantation for Coulomb blockade at room temperature," Appl. Surf. Sci., vol. 239, no. 1-4, pp. 132-137, Nov. 2004.

[7] J. Dufourcq, S. Bodnar, G. Gay, D. Lafond, P. Mur, G. Molas, J. P. Nieto, L. Vandroux, L. Jodin, F. Gustavo, and T. Baron, "High density platinum nanocrystals for non-volatile memory applications," Appl. Phys. Lett., vol. 92, no. 7, p. 073102, Feb. 2008.

[8] P. K. Singh, G. Bisht, R. Hofmann, K. Singh, N. Krishna, and S. Mahapatra, "Metal nanocrystal memory with Pt single- and dual-layer $\mathrm{NC}$ with low-leakage $\mathrm{Al}_{2} \mathrm{O}_{3}$ blocking dielectric," IEEE Electron Device Lett., vol. 29, no. 12, pp. 1389-1391, Dec. 2008.

[9] C. Sargentis, K. Giannakopoulos, A. Travlos, N. Boukos, and D. Tsamakis, "Simple method for the fabrication of a high dielectric constant metal-oxide-semiconductor capacitor embedded with Pt nanoparticles," Appl. Phys. Lett., vol. 88, no. 7, p. 073106, Feb. 2006.

[10] J. Carrey, J.-L. Maurice, F. Petroff, and A. Vaurès, "Growth of Au clusters on amorphous $\mathrm{Al}_{2} \mathrm{O}_{3}$ : Evidence of cluster mobility above a critical size," Phys. Rev. Lett., vol. 86, no. 20, pp. 4600-4603, May 2001.

[11] M. Hirasawa, H. Shirakawa, H. Hamamura, Y. Egashira, and H. Komiyama, "Growth mechanism of nanoparticles prepared by radio frequency sputtering," J. Appl. Phys., vol. 82, no. 3, pp. 1404-1407, Aug. 1997.

[12] L. Perniola, B. D. Salvo, G. Ghibaudo, A. F. Para, G. Pananakakis, V. Vidal, T. Baron, and S. A. Lombardo, "Modeling of the programming window distribution in multinanocrystals memories," IEEE Trans. Nanotechnol., vol. 2, no. 4, pp. 277-284, Dec. 2003.

[13] S. Huang, S. Banerjee, R. T. Tung, and S. Oda, "Electron trapping, storing, and emission in nanocrystalline $\mathrm{Si}$ dots by capacitance-voltage and conductance-voltage measurements," J. Appl. Phys., vol. 93, no. 1, pp. 576-581, Jan. 2003. 